\title{
DISPONIBILIDADE DE ZINCO PARA MILHO PELOS EXTRATORES MEHLICH-1, MEHLICH-3 E DTPA EM SOLOS DE MINAS GERAIS, NA PRESENÇA E AUSÊNCIA DE CALAGEM ${ }^{(1)}$
}

\author{
Agna Almeida Menezes ${ }^{(2)}$, Luiz Eduardo Dias ${ }^{(3)}$, Julio César Lima \\ Neves $^{(3)}$ \& Joelson Virginio Orrico da Silva ${ }^{(4)}$
}

\begin{abstract}
RESUMO
No Brasil, diversos extratores químicos são utilizados na determinação de Zn disponível para as plantas, destacando-se Mehlich-1 e DTPA. Este trabalho foi realizado com o objetivo de estudar a disponibilidade de $\mathrm{Zn}$ em solos de Minas Gerais em função de doses de Zn e de calcário e extratores químicos, utilizando-se milho como planta indicadora. Os extratores estudados foram: (a) Mehlich-1 com filtragem lenta após a extração (M-1f); (b) Mehlich-1 com retirada do sobrenadante após 16 h de repouco (M-1s); (c) Mehlich-3 (M-3); e (d) DTPA. As amostras receberam adubação básica com macro e micronutrientes (-Zn) e cinco doses de $\mathrm{Zn}$ na forma de $\mathrm{ZnSO}_{4}\left(0,2,46\right.$ e $8 \mathrm{mg} \mathrm{dm}^{-3}$ de $\left.\mathrm{Zn}\right)$ na ausência ou presença de calagem. Quinze dias após a fertilização, subamostras foram coletadas para a determinação de Zn pelos extratores. Para Mehlich-1, o extrato foi obtido por meio de dois procedimentos: filtragem logo após extração (M-1f) e retirada de alíquota após $16 \mathrm{~h}$ (M-1s). O restante do solo foi acondicionado em vasos plásticos, para o cultivo do milho durante 50 dias. A capacidade de extração variou na seguinte ordem: M-1s > M-1f > M-3 > DTPA, para todos os solos, na ausência e na presença de calagem. O M-1s e o M-3 não apresentaram diferenças na capacidade de extração com a calagem, enquanto M-1f e DTPA foram sensíveis à calagem. Os teores de Zn obtidos com todos os extratores correlacionaram-se significativa e negativamente com o teor de argila e a capacidade de campo dos solos, tanto na ausência como na presença de calagem. Os teores obtidos com DTPA apresentaram maior correlação com características de solo na presença de calagem, indicando que esse extrator aumentou sua sensibilidade à capacidade-tampão em valores mais elevados de
\end{abstract}

\footnotetext{
(1) Parte da Dissertação de Mestrado da primeira autora apresentada ao Programa de Solos e Nutrição de Plantas da Universidade Federal de Viçosa - UFV. Recebido para publicação em abril de 2009 e aprovado em janeiro de 2010.

${ }^{(2)}$ Professora do Departamento de Ciências Agrárias e Ambientais, Universidade Estadual de Santa Cruz - UESC. Rod. IlhéusItabuna, km 16, CEP 44650-000 Ilhéus (BA). E-mail: agna@uesc.br

(3) Professor do Departamento de Solos, Universidade Federal de Viçosa - UFV. CEP 36571-000 Viçosa (MG). E-mail: ledias@ufv.br

(4) Mestrando do Programa de Pós-Graduação em Produção Vegetal, UESC. E-mail: joelson.orrico@hotmail.com
} 
pH. Todos os extratores mostraram correlações com o conteúdo de $\mathrm{Zn}$ na planta; portanto, podem ser utilizados na avaliação da disponibilidade de Zn do solo.

Termos de indexação: micronutrientes, extrator, correlação, teor.

\author{
SUMMARY: ZINC AVAILABILITY TO MAIZE IN DIFFERENT SOILS WITH \\ AND WITHOUT LIMING DETERMINED BY MEHLICH-1, \\ MEHLICH-3 AND DTPA
}

\begin{abstract}
In Brazil, plant-available Zn in the soil is determined by several chemical procedures, of which the most commonly used are Mehlich-1 and DTPA. The objective of this study was to evaluate $Z n$ availability in soils of Minas Gerais State, under different $Z n$ and lime rates, using the extractors Mehlich-1, Mehlich-3 and DTPA and maize as indicator plant. Seven soil samples were incubated with lime to reach $p H$ 6.0. The samples were treated with $\mathrm{Zn}$ in the form of $\mathrm{ZnSO}_{4}$, in the absence or presence of lime, and with a basic Zn-free fertilization of macro and micronutrients. Fifteen days after fertilization, sub-samples were collected for Zn determination by the extractors. For Mehlich-1 the extract was obtained by two procedures: filtering soon after extraction (M-1f) and aliquot withdrawal from the supernatant after $16 \mathrm{~h}$ $(M-1 s)$. The remaining soil was filled into plastic pots for maize cultivation during 50 days. The extraction capacity diminished in the following order: $M-1 s>M-1 f>M-3>D T P A$, in all soils, with or without liming. M-1s and $M-3$ did not differ in extraction capacity in limed soil, whereas M-1f and DTPA were sensitive to liming. The $Z n$ concentration values obtained with all extractors were negative and significantly correlated with clay content and soil water field capacity, with or without liming. The extraction capacity of DTPA was better correlated with soil properties in the presence of lime, indicating that the response of this extractor is better in higher-pH soils. All extractors were correlated with plant Zn content, and can therefore be used in the evaluation of soil $\mathrm{Zn}$ availability.
\end{abstract}

Index terms: micronutrients, extractant, correlation, content.

\section{INTRODUÇÃO}

A análise química do solo, na avaliação da disponibilidade de Zn para as plantas, tem recebido grande destaque entre os métodos de diagnose, visto ser o único método que permite o conhecimento prévio dos teores disponíveis em locais onde serão implantadas culturas. No Brasil, esse destaque deve ser ainda maior, já que os solos, de maneira geral, são pobres em Zn. Além disso, a disponibilidade de Zn para as plantas pode ser reduzida devido a características do solo, como: $\mathrm{pH}$ elevado (Fornes et al., 2009; Lindsay, 1991), altos teores de argila (Shukla \& Mittal, 1979), de P (Barrow, 1987; Alloway, 1990), de C orgânico (Mandal \& Mandal, 1987; Stevenson, 1991; Uren, 1992), de óxidos de Fe, Al e Mn (McBride, 1989; Stahl \& James, 1991) e potencial redox (Gao et al., 2010).

As soluções extratoras mais comumente utilizadas para avaliar a disponibilidade de Zn são ácidas ou complexantes. No Brasil, o Mehlich-1, usado para avaliar a disponibilidade de $\mathrm{P}$ e $\mathrm{K}$, tem sido também utilizado para determinar a disponibilidade de alguns micronutrientes, como o Zn. A adoção desse extrator baseia-se no fato de fornecer extratos muito límpidos
(Raij, 1991) e teores recuperados, que se correlacionam significativamente com conteúdos de Zn em plantas (Evans et al., 1974; Couto, 1985; Galrão, 1995).

Dos extratores de Zn que agem por complexação, os mais comumente utilizados são soluções de EDTA e DTPA. Tanto o DTPA, proposto por Lindsay \& Norvell (1978), quanto o Mehlich-3, proposto por Mehlich (1984) e que possui EDTA em sua composição, foram idealizados como extratores para avaliar simultaneamente a disponibilidade de Zn, Fe, Mn e Cu e outros nutrientes.

Os extratores ácidos deslocam cátions adsorvidos, dissolvem carbonatos e hidróxidos não perfeitamente cristalizados e provocam dissolução parcial de minerais de argila silicatados (Pickering, 1981). Já os extratores quelatantes atuam no deslocamento de Zn de sítios de troca por meio da formação de complexos solúveis, reduzindo a atividade de íons em solução (Norvell, 1991) e sem alterar, significativamente, o $\mathrm{pH}$ dos solos, evitando, dessa maneira, a solubilização de compostos de Zn.

A seleção de um extrator na determinação da disponibilidade de um elemento no solo baseia-se no extrator-padrão: a planta. Dessa forma, Lantmann 
\& Meurer (1982) verificaram que a quantidade de Zn absorvida por plantas de milho em diferentes solos do Rio Grande do Sul, na ausência ou na presença de calagem, correlacionou-se melhor com os teores recuperados pelo extrator $\mathrm{Na}_{2}$ EDTA do que com os teores obtidos pelos extratores Mehlich-1 e $\mathrm{HCl}$ $0,1 \mathrm{~mol} \mathrm{~L}^{-1}$. Muraoka et al. (1983) e Ribeiro \& Tucunango Sarabia (1984) encontraram resultados semelhantes, quanto à avaliação da disponibilidade de Zn por esse extrator, para plantas de feijão e sorgo. Por outro lado, Mehlich-1 e DTPA apresentaram eficiência semelhante para a valiar a disponibilidade de Zn tanto para Coffea arabica L. como para $C$. canephora Pierre (Favarin et al., 2007).

A baixa disponibilidade de $\mathrm{Zn}$ de expressiva parcela de solos do Brasil, especialmente os de Cerrado, o efeito negativo da calagem sobre tal disponibilidade e as respostas positivas das plantas à fertilização com $\mathrm{Zn}$ ressaltam a importância da avaliação de sua disponibilidade no solo para obtenção de elevadas e sustentáveis produtividades das culturas. Este trabalho foi realizado com o objetivo de estudar a disponibilidade de $\mathrm{Zn}$ em solos de Minas Gerais em função de doses de Zn e de calcário e extratores químicos, utilizando-se milho como planta indicadora.

\section{MATERIAL E MÉTODOS}

Amostras da camada de $0-20 \mathrm{~cm}$ de sete solos de Minas Gerais foram destorroadas, peneiradas, secas ao ar e homogeneizadas individualmente. Em seguida, foram coletadas porções de cada amostra para a caracterização física e química (Quadro 1). De cada amostra de solo, foram obtidas 30 subamostras. Metade recebeu uma mistura de calcário dolomítico com $\mathrm{CaCO}_{3}$, na relação 4:1 de Ca:Mg, em dose necessária para elevar o $\mathrm{pH}\left(\mathrm{H}_{2} \mathrm{O}\right)$ para 6,0 , com base em curva de incubação. A mistura solo-corretivo foi homogeneizada, acondicionada em sacos plásticos e umedecida para alcançar $80 \%$ da água retida a $33 \mathrm{kPa}$. Procedimento semelhante foi adotado para a outra metade das subamostras, que não recebeu a mistura corretiva.

Após 10 dias de incubação, as subamostras foram secas ao ar e peneiradas $(4 \mathrm{~mm})$ para receberem as doses de $\mathrm{Zn}\left(0,2,4,6\right.$ e $\left.8 \mathrm{mg} \mathrm{dm}^{-3}\right)$, na forma de soluções de $\mathrm{ZnSO}_{4}$, que foram aplicadas e misturadas com todo o volume de solo da unidade experimental. Nessa mesma oportunidade, realizou-se adubação básica, com N (100 mg dm ${ }^{-3}$ na forma de $\mathrm{NH}_{4} \mathrm{NO}_{3} \mathrm{e}$ $\mathrm{NH}_{4} \mathrm{H}_{2} \mathrm{PO}_{4}$ ), $\mathrm{K}$ (para alcançar $150 \mathrm{mg} \mathrm{dm}^{-3} \mathrm{na}$ forma de $\left.\mathrm{KH}_{2} \mathrm{PO}_{4}\right), \mathrm{S}\left(80 \mathrm{mg} \mathrm{dm}^{-3}\right.$ na forma de $\left.\mathrm{MgSO}_{4}\right)$ e $\mathrm{P}$ (em dose baseada na classe de P-remanescente (P-rem) Alvarez V. et al. (2000), na forma de $\mathrm{NH}_{4} \mathrm{H}_{2} \mathrm{PO}_{4}$, $\mathrm{KH}_{2} \mathrm{PO}_{4}$ e $\mathrm{NaH}_{2} \mathrm{PO}_{4}$ ). Subamostras de cada unidade experimental foram retiradas 15 dias após a realização da adubação, com o objetivo de determinar os valores de $\mathrm{pH}$ e teores de $\mathrm{Zn}$ disponível pelos extratores Mehlich-1 (Embrapa, 1997), Mehlich-3 (Mehlich, 1984) e DTPA-TEA (Lindsay \& Norvell,1978). A dosagem de $\mathrm{Zn}$ nos extratos foi realizada por espectrofotometria de absorção atômica. Para o Mehlich-1, foram adotados dois procedimentos após extração: filtragem do tipo lenta logo após extração (M-1f) e retirada de alíquota do sobrenadante após $16 \mathrm{~h}$ de repouso (M-1s).

Quadro 1. Características físicas e químicas das amostras de solos utilizadas na condução do experimento

\begin{tabular}{|c|c|c|c|c|c|c|c|}
\hline $\begin{array}{c}\text { Solo }^{(1)} \\
\text { Procedência }\end{array}$ & $\begin{array}{c}\mathrm{LVd}_{1} \\
\text { Sete Lagoas }\end{array}$ & $\begin{array}{l}\text { LVAdf } \\
\text { Viçosa }\end{array}$ & $\begin{array}{c}\text { LVAw } \\
\text { Lassance }\end{array}$ & $\begin{array}{c}\text { LVAdh } \\
\text { Tiradentes }\end{array}$ & $\begin{array}{l}\text { PVAd }_{c} \\
\text { Ervália }\end{array}$ & $\begin{array}{c}\text { OY } \\
\text { Barbacena }\end{array}$ & $\begin{array}{c}\mathbf{L V d}_{2} \\
\text { Três Marias }\end{array}$ \\
\hline Argila $\left(\mathrm{g} \mathrm{kg}^{-1}\right)$ & 820 & 630 & 570 & 480 & 230 & 220 & 140 \\
\hline Silte $\left(\mathrm{g} \mathrm{kg}^{-1}\right)$ & 80 & 50 & 170 & 60 & 180 & 130 & 30 \\
\hline Areia $\left(\mathrm{g} \mathrm{kg}^{-1}\right)$ & 100 & 320 & 260 & 460 & 590 & 650 & 830 \\
\hline $\mathrm{AR}\left(\mathrm{g} \mathrm{kg}^{-1}\right)$ & 330 & 280 & 260 & 220 & 190 & 590 & 70 \\
\hline $\mathrm{C}$ org. $\left(\mathrm{g} \mathrm{kg}^{-1}\right)$ & 26,9 & 33,9 & 2,3 & 24,9 & 6,6 & 385,7 & 4,3 \\
\hline $\mathrm{pH}^{(4)}$ & 4,6 & 4,5 & 5,0 & 4,9 & 5,1 & 4,8 & 4,8 \\
\hline $\mathrm{Ca}^{2+}\left(\mathrm{cmol}_{\mathrm{c}} \mathrm{dm}^{-3}\right)^{(5)}$ & 1,4 & 0,3 & 0,5 & 1,1 & 2,1 & 4,4 & 0,1 \\
\hline $\mathrm{Mg}^{2+}\left(\mathrm{cmol}_{\mathrm{c}} \mathrm{dm}^{-3}\right)^{(5)}$ & 0,8 & 0,6 & 0,6 & 1,7 & 1,3 & 1,4 & 0,5 \\
\hline $\mathrm{Al}^{3+}\left(\mathrm{cmol}_{\mathrm{c}} \mathrm{dm}^{-3}\right)^{(5)}$ & 1,4 & 0,3 & 0,7 & 1,0 & 0,0 & 0,8 & 0,2 \\
\hline $\mathrm{H}+\mathrm{Al}\left(\mathrm{cmol}_{\mathrm{c}} \mathrm{dm}^{-3}\right)^{(6)}$ & 9,6 & 11,4 & 3,3 & 10,2 & 3,3 & 22,2 & 3,3 \\
\hline $\mathrm{t}\left(\mathrm{cmol}_{\mathrm{c}} \mathrm{dm}^{-3}\right)$ & 3,7 & 1,3 & 1,9 & 2,9 & 3,5 & 6,8 & 0,9 \\
\hline $\mathrm{T}\left(\mathrm{cmol}_{\mathrm{c}} \mathrm{dm}^{-3}\right)$ & 11,9 & 12,4 & 4,5 & 12,1 & 6,8 & 28,1 & 4,0 \\
\hline $\mathrm{P}\left(\mathrm{mg} \mathrm{dm} \mathrm{m}^{-3}\right)^{(7)}$ & 3,8 & 3,6 & 3,0 & 2,1 & 8,2 & 146,7 & 2,2 \\
\hline 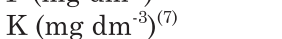 & 35 & 27 & 44 & 23 & 63 & 58 & 21 \\
\hline $\mathrm{Zn}\left(\mathrm{mg} \mathrm{dm}{ }^{-3}\right)^{(7)}$ & 0,58 & 0,45 & 0,38 & 0,70 & 3,47 & 5,99 & 0,24 \\
\hline P-rem $\left(\mathrm{mg} \mathrm{L}^{-1}\right)^{(8)}$ & 6,06 & 7,17 & 0,37 & 6,06 & 46,65 & 5,45 & 45,68 \\
\hline
\end{tabular}

(1) Classes de solos: $\mathrm{LVd}_{1}$ : Latossolo Vermelho distrófico típico - argiloso; LVAdf: Latossolo Vermelho-Amarelo distroférrico típico; LVAw: Latossolo Vermelho-Amarelo ácrico; LVAh: Latossolo Vermelho-Amarelo distrófico húmico; PVAd : Argissolo VermelhoAmarelo distrófico câmbico; OY: Organossolo Mésico ; $\mathrm{LVd}_{2}$ : Latossolo Vermelho distrófico textura média. (2) Água retida a -33 kPa. ${ }^{(3)}$ Método Walkley-Black. ${ }^{(4)} \mathrm{pH}$ em água relação solo:solução $1: 2,5 .{ }^{(5)} \mathrm{KCl} 1 \mathrm{~mol} \mathrm{~L}^{-1}$. $\left.{ }^{(6)} \mathrm{Ca}^{(\mathrm{OAc})}\right)_{2} 0,5 \mathrm{mg} \mathrm{L}^{-1} \mathrm{pH} 7,0$.

(7) Mehlich-1. ${ }^{(8)}$ Solução de $60 \mathrm{mg} \mathrm{L}^{-1}$ de P. 
Em seguida, as amostras de solo foram acondicionadas em vasos plásticos, para plantio de milho híbrido BR 201. Durante o período de cultivo, as amostras de solo receberam, a cada 15 dias, a aplicação de $\mathrm{N}\left(25 \mathrm{mg} \mathrm{dm}^{-3}\right.$ na forma de $\left.\mathrm{NH}_{4} \mathrm{NO}_{3}\right) \mathrm{e}$ uma solução de micronutrientes (Alvarez V. et al., 1983), sem Zn.

O experimento foi conduzido em casa de vegetação, com os tratamentos em esquema fatorial $7 \times 2 \times 5$, correspondendo a sete solos, com ou sem calagem, e cinco doses de Zn, distribuídos em blocos casualizados com três repetições.

Aos 50 dias de cultivo, em casa de vegetação, a parte aérea das plantas foi cortada a $1 \mathrm{~cm}$ acima do solo, seca em estufa a $70^{\circ} \mathrm{C}$ por $48 \mathrm{~h}$, pesada e moída. Os teores de $\mathrm{Zn}$ no material vegetal foram determinados por digestão nítrico-perclórica, e a dosagem dos extratos, por espectrofotometria de absorção atômica.

Os teores de $\mathrm{Zn}$ recuperados pelos extratores Mehlich-1, Mehlich-3 e DTPA, os teores e conteúdos de Zn na parte aérea das plantas e a massa da parte aérea seca foram submetidos à análise de variância, sendo o efeito de dose desdobrado, para cada solo e extrator na presença ou ausência de calagem, por meio de análise de regressão. Foram obtidas duas equações, para cada solo e extrator em que foram comparadas, mediante teste de identidade de modelos, utilizando o procedimento Regrelin do software SAEG 5.0, de modo a inferir a sensibilidade dos extratores à calagem. As declividades das equações do $\mathrm{Zn}$ recuperado em função do adicionado foram correlacionadas com algumas características dos solos, objetivando verificar a sensibilidade dos extratores a outras características que refletem a capacidade-tampão. Para cada solo e dose de calagem, foram ajustadas equações de regressão para os teores e os conteúdos de Zn na planta, como variáveis dependentes das doses de Zn adicionadas. As duas equações assim obtidas foram comparadas mediante teste de identidade de modelos, visando avaliar a sensibilidade da planta, em termos da absorção de Zn, à calagem.

\section{RESULTADOS E DISCUSSÃO}

Os teores de Zn obtidos pelos extratores M-1, M-3 e DTPA aumentaram linearmente com as doses de $\mathrm{Zn}$ adicionadas, para todos os solos, tanto na ausência como na presença de calagem. A recuperação de Zn variou com os solos, com os extratores e, para alguns destes, com a calagem, como indicado pelas declividades das equações ajustadas (Quadro 2). Em termos médios, a recuperação de Zn, considerando todos os solos e a calagem, obedeceu à sequência: M-1 $>$ M-3 > DTPA. Apesar de uma clara tendência para valores de declividade menores para as amostras que receberam a mistura corretiva, para M-1s e M-3 as equações não diferiram em função da calagem; por isso, uma única equação representa o relacionamento para cada solo (Quadro 2). O M-3, contudo, mostrou menor capacidade de recuperação de que o Mehlich-1 em todos os solos, como indicado pelas menores declividades das equações. Considerando a média das declividades para o M-3 $(0,54)$ e para o Mehlich-1 $(0,66)$, a recuperação de $\mathrm{Zn}$ pelo primeiro foi 18,3 \% menor que a obtida pelo segundo.

O DTPA, que apresentou a menor capacidade de recuperação de $\mathrm{Zn}$ entre os extratores estudados, foi sensível à elevação de $\mathrm{pH}$ provocada pela calagem. Essa sensibilidade foi maior do que a do M-1f, provavelmente pelo fato de o DTPA ter sido sensível à calagem em maior número de solos, além do maior decréscimo na recuperação de Zn (20,7 \%) comparado ao M-1f (13,0 \%) na presença de calagem, considerando apenas os solos em que os referidos extratores foram sensíveis à calagem. Resultados semelhantes foram obtidos por Abreu \& Raij (1997) e Nascimento \& Fontes (2004).

Como era de se esperar, o teor de argila e água retida a $-33 \mathrm{kPa}$ apresentaram correlações significativas e negativas com as declividades dos modelos de $\mathrm{Zn}$ recuperado pelos extratores em função do adicionado (Quadro 3). Além dessas características, as correlações significativas com o $\mathrm{P}$ remanescente, também verificadas por Couto et al. (1985), sugerem que essas características podem ser utilizadas como critério auxiliar na avaliação da disponibilidade e na recomendação de fertilização com Zn, notadamente para extratores sensíveis à capacidade-tampão de $\mathrm{Zn}$ no solo.

M-1s e M-3 não foram sensíveis à calagem, porém a disponibilidade de Zn pelo M-3 mostrou melhor correlação com todas as características de solos que o M-1s. Para o Mehlich-1, porém, a filtragem logo após extração (M-1f) resultou em aumento nos valores dos coeficientes de correlação e no número de correlações significativas. A elevação do $\mathrm{pH}$ do solo também provocou modificações na sensibilidade do DTPA-TEA à capacidade-tampão de $\mathrm{Zn}$. Na ausência de calagem, este extrator apresentou menor sensibilidade à capacidade-tampão. No entanto, com a elevação do $\mathrm{pH}$, o extrator apresentou-se mais bem correlacionado com todas as características dos solos que os demais.

A falta de sensibilidade ao aumento de $\mathrm{pH}$ do solo pelo extrator Mehlich-3 foi também verificada por Junus \& Cox (1987), quando avaliaram a disponibilidade de $\mathrm{Zn}$ por esse extrator em oito solos numa faixa de variação do $\mathrm{pH}$ de 4,5 a 7,1. Esse fato pode ser explicado com base nos diferentes mecanismos de extração, resultantes da composição química dos extratores. Assim, o Mehlich-1, constituído por dois ácidos fortes diluídos e com $\mathrm{pH}$ em torno de 1,2 , extrai por dissolução ácida e possui maior capacidade de solubilização de formas precipitadas de $\mathrm{Zn}$ do que o $\mathrm{M}-3$, que é menos ácido ( $\mathrm{pH}$ 2,5) e extrai $\mathrm{Zn}$ por complexação e por troca com íons $\mathrm{NH}_{4}{ }^{+}$(Tran \& 
Quadro 2. Equações, obtidas por análise de regressão, para os teores $\left(\mathrm{mg} \mathrm{dm}^{-3}\right)$ de zinco recuperado pelos extratores Mehlich-1, Mehlich-3 e DTPA-TEA em função das doses $\left(\mathrm{mg} \mathrm{dm}^{-3}\right)$ de zinco adicionadas, na ausência ou presença de calagem

\begin{tabular}{|c|c|c|c|c|c|c|c|c|c|c|}
\hline \multirow{2}{*}{ Solo } & \multicolumn{3}{|c|}{ Cal 0} & \multicolumn{3}{|c|}{ Cal 1} & \multirow{2}{*}{ Sig. } & \multicolumn{3}{|c|}{ Equação conjunta } \\
\hline & $b_{o}$ & $\mathbf{b}_{1}^{* *}$ & $\mathbf{R}^{2}$ & $b_{o}$ & $b_{1}{ }^{* * *}$ & $\mathrm{R}^{2}$ & & $b_{o}$ & $b_{1}^{* *}$ & $\overline{\mathrm{R}^{2}}$ \\
\hline \multicolumn{11}{|c|}{ Mehlich-1 (sobrenadante) } \\
\hline $\operatorname{LVd}_{1}$ & 0,53 & 0,6775 & 0,996 & 0,73 & 0,5905 & 0,999 & \# & 0,66 & 0,6528 & 0,991 \\
\hline LVAdf & 0,50 & 0,7820 & 0,995 & 0,63 & 0,7090 & 0,995 & \# & 0,62 & 0,7609 & 0,996 \\
\hline LVAw & 0,53 & 0,6925 & 0,991 & 0,61 & 0,6525 & 0,992 & \# & 0,59 & 0,6953 & 0,991 \\
\hline LVAdh & 0,83 & 0,7595 & 0,999 & 0,82 & 0,7175 & 0,999 & \# & 0,88 & 0,7556 & 0,997 \\
\hline PVAdc & 4,82 & 0,8575 & 0,965 & 4,85 & 0,7180 & 0,998 & \# & 5,14 & 0,7237 & 0,937 \\
\hline OY & 6,39 & 0,6895 & 0,965 & 6,05 & 0,5960 & 0,989 & \# & 6,36 & 0,6331 & 0,927 \\
\hline $\mathrm{LVd}_{2}$ & 0,34 & 0,8695 & 0,989 & 0,53 & 0,7715 & 0,996 & $\#$ & 0,45 & 0,8441 & 0,987 \\
\hline \multicolumn{11}{|c|}{ Mehlich-1 (filtrado) } \\
\hline $\mathrm{LVd}_{1}$ & 0,38 & 0,5820 & 0,999 & 0,40 & 0,4770 & 0,999 & & & & \\
\hline LVAdf & 0,39 & 0,6900 & 0,999 & 0,53 & 0,5810 & 0,998 & & & & \\
\hline LVAw & 0,69 & 0,5735 & 0,995 & 0,56 & 0,6045 & 0,990 & \# & 0,66 & 0,5990 & 0,992 \\
\hline LVAdh & 0,79 & 0,6645 & 0,995 & 0,59 & 0,6275 & 0,993 & & & & \\
\hline PVAdc & 4,56 & 0,7220 & 0,907 & 4,01 & 0,6905 & 0,977 & \# & 4,36 & 0,6857 & 0,913 \\
\hline OY & 5,43 & 0,5250 & 0,981 & 4,77 & 0,6020 & 0,998 & \# & 5,19 & 0,5416 & 0,975 \\
\hline $\mathrm{LVd}_{2}$ & 0,29 & 0,7905 & 0,989 & 0,37 & 0,7415 & 0,999 & \# & 0,37 & 0,7683 & 0,994 \\
\hline \multicolumn{11}{|c|}{ Mehlich-3 } \\
\hline $\mathrm{LVd}_{1}$ & 0,22 & 0,4675 & 0,988 & 0,32 & 0,4275 & 0,998 & \# & 0,24 & 0,4667 & 0,991 \\
\hline LVAdf & 0,34 & 0,4980 & 0,994 & 0,49 & 0,4515 & 0,980 & \# & 0,30 & 0,5118 & 0,992 \\
\hline LVAw & 0,29 & 0,5215 & 0,990 & 0,26 & 0,5155 & 0,996 & \# & 0,23 & 0,5339 & 0,995 \\
\hline LVAdh & 0,63 & 0,4525 & 0,971 & 0,42 & 0,5105 & 0,998 & \# & 0,76 & 0,4158 & 0,840 \\
\hline PVAdc & 4,29 & 0,7100 & 0,995 & 4,01 & 0,6890 & 0,985 & \# & 4,31 & 0,6758 & 0,932 \\
\hline OY & 3,39 & 0,4370 & 0,993 & 3,43 & 0,3960 & 0,967 & \# & 3,48 & 0,3889 & 0,973 \\
\hline $\mathrm{LVd}_{2}$ & 0,29 & 0,7830 & 0,992 & 0,33 & 0,7850 & 0,999 & \# & 0,31 & 0,7866 & 0,997 \\
\hline \multicolumn{11}{|c|}{ DTPA-TEA } \\
\hline $\mathrm{LVd}_{1}$ & 0,26 & 0,4625 & 0,997 & 0,24 & 0,3500 & 0,998 & & & & \\
\hline LVAdf & 0,34 & 0,5835 & 0,998 & 0,33 & 0,4495 & 0,997 & & & & \\
\hline LVAw & 0,53 & 0,4325 & 0,998 & 0,34 & 0,4470 & 0,992 & \# & 0,45 & 0,4413 & 0,993 \\
\hline LVAdh & 0,53 & 0,5765 & 0,999 & 0,42 & 0,4160 & 0,999 & & & & \\
\hline PVAdc & 3,44 & 0,5300 & 0,999 & 3,08 & 0,5590 & 0,993 & \# & 3,36 & 0,5694 & 0,895 \\
\hline OY & 3,78 & 0,4490 & 0,997 & 3,22 & 0,4060 & 0,992 & & & & \\
\hline $\mathrm{LVd}_{2}$ & 0,27 & 0,6960 & 0,993 & 0,37 & 0,5745 & 0,995 & & & & \\
\hline
\end{tabular}

Classes de solos: $\mathrm{LVd}_{1}$ : Latossolo Vermelho distrófico típico argiloso; LVAdf: Latossolo Vermelho-Amarelo distroférrico típico;

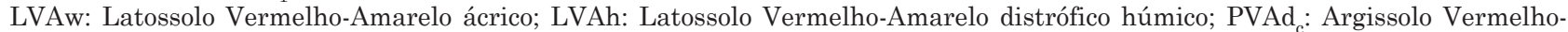
Amarelo distrófico câmbico; OY: Organossolo Mésico; $\mathrm{LVd}_{2}$ : Latossolo Vermelho distrófico textura média; \#: equações que não diferem entre si pelo teste de identidade de modelos, de forma que foi adotada apenas a equação conjunta; **: significativo a $1 \%$ pelo teste $\mathrm{F}$; $\mathrm{b}_{\mathrm{o}}$ : intercepto $\left(\mathrm{mg} \mathrm{\textrm {dm } ^ { - 3 } )}\right.$; $\mathrm{b}_{1}$ : coeficiente linear $\left(\mathrm{mg} \mathrm{dm}^{-3}\right)$.

Simard, 1993). Oliveira et al. (1999), estudando a relação entre o $\mathrm{Zn}$ disponível pelos extratores $\mathrm{HCl}$, Mehlich-1, Mehlich-3 e DTPA-TEA e as frações Zn trocável e Zn ligado à matéria orgânica, a óxidos de Mn, a óxidos de Fe amorfo e a óxidos de Fe cristalino, verificaram que a fração $\mathrm{Zn}$ trocável foi a maior responsável pelo teor de $\mathrm{Zn}$ obtido pelos extratores, mas ressaltam que as frações estudadas não foram suficientes para explicar, em todos os casos, o $\mathrm{Zn}$ recuperado pelos extratores.

A filtragem modificou a sensibilidade do Mehlich-1 à capacidade-tampão de Zn; já o DTPA-TEA, na ausência de calagem, apresentou menor sensibilidade à capacidade-tampão, mas, com a elevação do $\mathrm{pH}$, ficou mais bem correlacionado com todas as características dos solos que os demais, sugerindo maior eficiência em solos com maior $\mathrm{pH}$. Norvell (1984) recomenda o DTPA-TEA para solos com maiores valores de $\mathrm{pH}$, advertindo, inclusive, que o uso dessa solução extratora em solos ácidos resulta na diminuição do tamponamento do extrator.

O teste de identidade de modelos demonstrou maior sensibilidade do DTPA-TEA à calagem. Muraoka et al. (1983) verificaram redução no teor de Zn na parte aérea de plantas de feijão, em resposta à calagem, quando o $\mathrm{pH}$ do solo passou, em média, de 5,5 para 6 ,8. Para milho, Junus \& Cox (1987) constataram redução média de $42 \%$ no teor de Zn na parte aérea das plantas com o aumento de duas unidades de $\mathrm{pH}$ do solo $(4,5$ a 6,5$)$. A diminuição na absorção de $\mathrm{Zn}$ 
promovida pela calagem sugere diminuição da disponibilidade do elemento, devido ao aumento da adsorção de $\mathrm{Zn}$ no solo com o incremento de $\mathrm{pH}$, ou mesmo diminuição da solubilidade do elemento na solução do solo. Para plantas de trigo, Sims (1986) constatou que a absorção de $\mathrm{Zn}$ diminuiu com o aumento do $\mathrm{pH}$ do solo de 4,5 para 5,8 e atribuiu esse efeito também à maior adsorção no solo, mostrando que formas trocáveis de $\mathrm{Zn}$ foram transformadas em formas ligadas à matéria orgânica, menos disponível para plantas. Resultados semelhantes foram também observados por Iyengar et al. (1981) e Sims \& Patrick (1978).

Os teores e conteúdos de Zn na parte aérea das plantas de milho variaram com o solo, com a dose e com a calagem. Com exceção das amostras do solo OY, para as demais houve aumento linear na absorção de $\mathrm{Zn}$ (conteúdo) em função das doses de $\mathrm{Zn}$ adicionadas (Quadro 4). O resultado do teste de identidade entre os modelos ajustados mostrou que, para todos os solos, as equações foram diferentes, indicando sensibilidade das plantas à calagem. As menores declividades das equações na presença de

Quadro 3. Coeficientes de correlação linear entre as declividades da equação de $\mathrm{Zn}$ adicionado e recuperado pelos extratores Mehlich-1, Mehlich-3 e DTPA-TEA, com algumas características dos solos que refletem o fator capacidade

\begin{tabular}{|c|c|c|c|c|c|c|}
\hline \multirow{2}{*}{ Característica de solo } & \multirow{2}{*}{ M-1s } & \multicolumn{2}{|c|}{ M-1f } & \multirow{2}{*}{ M-3 } & \multicolumn{2}{|c|}{ DTPA-TEA } \\
\hline & & Cal 0 & Cal 1 & & Cal 0 & Cal 1 \\
\hline Argila & $-0,743^{*}$ & $-0,819^{*}$ & $-0,971^{* *}$ & $-0,830^{*}$ & $-0,736^{*}$ & $-0,942^{*}$ \\
\hline$\Psi_{-33}$ & $-0,861^{*}$ & $-0,871^{*}$ & $-0,948^{* *}$ & $-0,831^{*}$ & $-0,797^{*}$ & $-0,837^{*}$ \\
\hline Corgânico & $-0,199^{\mathrm{ns}}$ & $-0,208^{\mathrm{ns}}$ & $-0,622^{\circ}$ & $-0,690^{\circ}$ & $-0,023^{\mathrm{ns}}$ & $-0,644^{\circ}$ \\
\hline $\mathrm{Al}^{3+}$ & $-0,610^{\circ}$ & $-0,744^{*}$ & $-0,781^{*}$ & $-0,756^{*}$ & $-0,620^{\circ}$ & $-0,920$ ** \\
\hline $\mathrm{H}+\mathrm{Al}$ & $-0,192^{\mathrm{ns}}$ & $-0,242^{\mathrm{ns}}$ & $-0,619^{\circ}$ & $-0,760^{*}$ & $-0,051^{\mathrm{ns}}$ & $-0,702^{\circ}$ \\
\hline CTC & $0,323^{\mathrm{ns}}$ & $-0,312^{\mathrm{ns}}$ & $-0,656^{\mathrm{ns}}$ & $-0,772^{*}$ & $-0,106^{\mathrm{ns}}$ & $-0,676^{\circ}$ \\
\hline $\mathrm{P}$ rem $^{(60)}$ & $0,548^{\mathrm{ns}}$ & $0,735^{*}$ & $0,758^{*}$ & $0,896^{* *}$ & $0,6710^{\circ}$ & $0,897^{*}$ \\
\hline
\end{tabular}

M-1s: retirada de alíquota após 16 h; M-1f: filtragem logo após extração; M-3: Mehlich-3; DTPA-TEA: DTPA-TEA-TEA; $\Psi_{-33}$ : água retida no solo a $-33 \mathrm{kPa} ;{ }^{* *},{ }^{*},{ }^{\circ}$, ns: significativo a $1,5,10 \%$ e não significativo, respectivamente.

Quadro 4. Equações de regressão para os teores ( $\left.\mathrm{mg} \mathrm{kg}^{-1}\right)$ e conteúdos (mg/vaso) de zinco na parte aérea de plantas de milho, em função de doses de zinco adicionadas, na ausência e presença de calagem

\begin{tabular}{|c|c|c|c|c|c|c|c|c|c|}
\hline \multirow{2}{*}{ Solo } & \multicolumn{4}{|c|}{ Cal 0} & \multicolumn{4}{|c|}{ Cal 1} & \multirow{2}{*}{ Sig. } \\
\hline & $\mathbf{b}_{\mathrm{o}}$ & $\mathbf{b}_{1}^{* * *}$ & $b_{11}{ }^{*}$ & $\mathbf{R}^{2}$ & $\mathbf{b}_{\mathrm{o}}$ & $b_{1}^{* * *}$ & $b_{11}$ & $\mathbf{R}^{2}$ & \\
\hline \multicolumn{10}{|c|}{ Teor } \\
\hline $\mathrm{LVd}_{1}$ & 11,7 & 4,6675 & - & 0,948 & 11,3 & 2,1000 & - & 0,967 & $\mathrm{x}$ \\
\hline LVAdf & 15,2 & 4,7400 & - & 0,982 & 8,6 & 2,5008 & - & 0,983 & $\mathrm{x}$ \\
\hline LVAw & 15,3 & 4,5017 & - & 0,921 & 8,9 & 3,9517 & - & 0,987 & $\mathrm{x}$ \\
\hline LVAdh & 13,1 & 4,6958 & - & 0,993 & 9,2 & 1,7542 & - & 0,978 & $\mathrm{x}$ \\
\hline PVAdc & 33,9 & 4,6033 & - & 0,973 & 33,1 & 3,3650 & - & 0,978 & $\mathrm{x}$ \\
\hline OY & 21,1 & 1,2558 & - & 0,628 & 22,7 & - & - & - & $\mathrm{x}$ \\
\hline \multicolumn{10}{|c|}{ Conteúdo } \\
\hline $\operatorname{LVd}_{1}$ & 144,0 & 65,31 & - & 0,927 & 139,1 & 37,97 & - & 0,952 & $\mathrm{x}$ \\
\hline LVAdf & 193,9 & 64,61 & - & 0,930 & 128,7 & 40,39 & - & 0,966 & $\mathrm{x}$ \\
\hline LVAw & 136,3 & 39,70 & - & 0,907 & 77,7 & 38,53 & - & 0,979 & $\mathrm{x}$ \\
\hline LVAdh & 176,3 & 68,24 & - & 0,997 & 129,8 & 31,16 & - & 0,995 & $\mathrm{x}$ \\
\hline PVAdc & 465,5 & 62,77 & & 0,900 & 432,8 & 43,84 & - & 0,993 & $\mathrm{x}$ \\
\hline OY & 276,8 & 72,61 & $-7,486$ & 0,882 & 298,4 & - & - & - & \\
\hline $\mathrm{LVd}_{2}$ & & & & & & & & & \\
\hline
\end{tabular}

Classes de solos: $\mathrm{LVd}_{1}$ : Latossolo Vermelho distrófico típico argiloso; LVAdf: Latossolo Vermelho-Amarelo distroférrico típico;

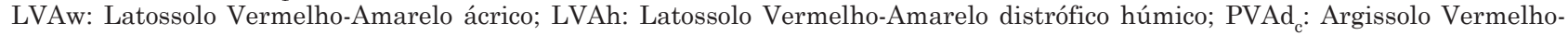
Amarelo distrófico câmbico; OY: Organossolo Mésico ; $\mathrm{LVd}_{2}$ : Latossolo Vermelho distrófico textura média; x: equações que diferem entre si pelo teste de identidade de modelos. ${ }^{* *},{ }^{*}$ : significativo a 1 e $5 \%$; coeficientes dos parâmetros das regressões - $b_{0}$ : intercepto; $b_{1}$ : coeficiente linear; e $b_{11:}$ coeficente quadrático. 
calagem mostram redução na absorção. Considerando a média das declividades das equações significativas para teor e conteúdo em função das doses de Zn, na presença e ausência de calagem, a aplicação do calcário resultou na redução média de 41,2 \% nos teores e de $36,2 \%$ nos conteúdos.

A menor absorção de $\mathrm{Zn}$ decorrente da calagem não refletiu em drástica diminuição de produção de biomassa, uma vez que a média dos valores de matéria seca para todos os solos, exceto OY, na ausência e presença de calagem apresentou valores muito próximos: 12,5 e 13,5 g/vaso, respectivamente.

A calagem, além de promover diminuição nos teores de $\mathrm{Zn}$, modificou a sensibilidade das plantas à capacidade-tampão de Zn nos diferentes solos. Com base nos coeficientes de variação das declividades das equações entre os teores de Zn nas plantas em função das doses de Zn, considerando somente os solos que apresentaram equações significativas na presença e na ausência de calagem, o CV na presença de calagem foi de $33 \%$, enquanto na ausência esse valor foi igual a $2 \%$.

\section{CONCLUSÕES}

1. A capacidade de extração de $\mathrm{Zn}$ variou na seguinte ordem: M-1s > M-1f $>$ M-3 > DTPA, para todos os solos, na ausência e na presença de calagem.

2. Os extratores M-1s e M-3 não apresentaram diferenças na capacidade de extração com a calagem, enquanto o M-1f e o DTPA foram sensíveis à calagem.

3. Os teores de $\mathrm{Zn}$ de todos os extratores correlacionaram-se significativa e negativamente com o teor de argila e a capacidade de campo dos solos, tanto na ausência como na presença de calagem. No entanto, os teores disponíveis com DTPA, nas amostras que receberam calagem, apresentaram maior correlação com essas características de solo.

4. Os teores disponíveis de $\mathrm{Zn}$ obtidos com os extratores correlacionaram-se significativamente com os conteúdos de Zn nas plantas, mostrando, portanto, a viabilidade da utilização destes para a avaliação da disponibilidade de $\mathrm{Zn}$ do solo.

\section{LITERATURA CITADA}

ABREU, C.A. \& RAIJ, B.van. Efeito da reação do solo no zinco extraído pelas soluções de DTPA e Mehlich-1. In: CONGRESSO BRASILEIRO DE CIÊNCIA DO SOLO, 26., Rio de Janeiro, 1997. Anais. Rio de Janeiro, Sociedade Brasileira de Ciência do Solo, 1997. CD-ROM.

ALLOWAY, B.J. The origins of heavy metals in soils. In: ALLOWAY, B.J., ed. Heavy metals in soils. New York, John Wiley \& Sons, 1990. p.29-39.
ALVAREZ V., V.H.; NOVAIS, R.F.; DIAS, L.E. \& OLIVEIRA, J.A. Determinação e uso de fósforo remanescente. B. Inf. SBCS, 25:27-32, 2000.

ALVAREZ V., H.V.; NOVAIS, R.F.; NEVES, J.C.L. \& BARROS, N.F. Níveis críticos de enxofre em solos de Cerrado para o crescimento de mudas de eucalipto. In: CONGRESSO BRASILEIRO DE CIÊNCIA DO SOLO, 19., Curitiba, 1983. Resumos. Curitiba, Sociedade Brasileira de Ciência do Solo. p.164.

BARROW, N.J. The effect of phosphate on zinc sorption by a soil. J. Soil Sci., 38:453-459, 1987.

COUTO, C. Resposta do eucalipto e do milho à aplicação de zinco em amostras de solos de Cerrado. Viçosa, MG, Universidade Federal de Viçosa, 1985. 97p. (Tese de Mestrado)

EMPRESA BRASILEIRA DE PESQUISA AGROPECUÁRIA . EMBRAPA. Centro Nacional de Pesquisa de Solos. Manual de métodos de análises de solos. 2.ed. Rio de Janeiro, 1997. 212p.

EVANS, C.C.; WEAR, J.J.; HAJEK, B.F. \& COPER, J.T. The relationship of soil zinc removed by three extractants to zinc uptake by corn and sorghum in medium - to - fine textured soils. Comm. Soil Sci. Plant Anal., 5:105-113, 1974.

FAVARIN, J.L.; VITTI, G.C.; DOURADO NETO, D.; FAVARIN JR, J.L. \& SALGADO, P.R. Teor de zinco no café como variável da disponibilidade no solo e extrator. R. Bras. Ci Solo, 31:1191-1196, 2007.

FORNES, F.; GARCIA-DE-LA-FUENTE, R.; BELDA, R.M. \& ABAD, M. "Alperjo" compost amendment of contaminated calcareous and acidic soils: effects on growth and trace element uptake by Five Brassica species. Bioresource Technol., 100:3982-3990. 2009.

GALRÃO, E.Z. Níveis críticos de zinco para o milho cultivado em Latossolo Vermelho-Amarelo, fase Cerrado. R. Bras. Ci. Solo, 19:255-260, 1995.

GAO, X.; SCHRÖDER, T.; HOFFLAND, E.; ZOU, C.; ZHANG, F. \& ZEE, S.E.A.T.M. van. Geochemical modeling of zinc availability for rice. Soil Sci. Soc. Am. J., 74:301-309. 2009.

IYENGAR, S S.; MARTENS, D. C. \& MILLER, W.P. Distribution and plant availability of soil zinc fractions. Soil Sci. Soc. Am. J., 45:735-739, 1981.

JUNUS, M.A. \& COX, F.R. A zinc soil test calibration based upon Mehlich-3 extractable zinc, $\mathrm{pH}$, and cation exchange capacity. Soil Sci. Soc. Am. J., 51:678-683, 1987.

LANTMANN, A.F. \& MEURER, E.J. Estudo da eficiência de extratores para avaliação do zinco disponível do solo para o milho. R. Bras. Ci. Solo, 16:131-135, 1982.

LINDSAY, W.L. \& NORVELL, W.A. Development of a DTPA soil test for zinc, iron, manganese, and copper. Soil Sci. Soc. Am. J., 42:421-428, 1978.

LINDSAY, W.L. Inorganic equilibria affecting micronutriens. In: MORTVEDT, J.J.; COX, F.R.; SHUMAN, L.M. \& WELCH, R.M., eds. Micronutrients in agriculture. 2.ed. Winsconsin, Soil Science Society of America, 1991. p.89111. 
McBRIDE, M.B. Surface chemistry of soil minerals. In: DIXON, J.B. \& WEED, S.B., eds. Minerals in soil environments. Madison, Soil Science Society of America, 1989. p.35-84.

MANDAL, L.N. \& MANDAL, B. Transformation of zinc fractions in rice soils. Soil Sci., 143:205-212, 1987.

MEHLICH, A. Mehlich-3 soil test extractant: A modification of Mehlich 2 extractants. Comm. Soil Sci. Plant Anal., 15:1409-1416, 1984.

MURAOKA, T.; NEPTUNE, A.M.L. \& NASCIMENTO FILHO, V.F. Avaliação da disponibilidade de zinco e de manganês do solo para o feijoeiro. I. Zinco. R. Bras. Ci. Solo, 7:167$175,1983$.

NASCIMENTO, C.W.A. \& FONTES, R.L.F. Correlação entre características de Latossolos e parâmetros de equações de adsorção de cobre e zinco. R. bras. Ci. Solo, 28:965-971. 2004.

NORVELL, W.A. Comparison of chelating agents as extractants for metals in diverse soil materials. Soil Sci. Sci. Am. J., 48:1285-1292, 1984.

NORVELL, W.A. Reactions of metal chelates in soils and nutrient solutions. In: MORTVEDT, J.J.; COX, F.R.; SHUMAN, L.M. \& WELCH, R.M., eds. Micronutrients in agriculture. Madison, Soil Science Society of America, 1991. p.187-228.

OLIVEIRA, M.F.G.; NOVAIS, R.F.; \& NEVES, J.C.L. Relação entre o zinco disponível, por diferentes extratores, e as frações de zinco em amostras de solos. R. Bras. Ci. Solo, 23:827-836, 1999.

PICKERING, W.F. Selective chemical extraction of soil components and bound metal species. Crit. Rev. Anal. Chem., 12:233-266, 1981.
RAIJ, B.van. Fertilidade do solo e adubação. São Paulo, Agronômica Ceres, 1991. 108p.

RIBEIRO, A.C. \& TUCUNANGO SARABIA, W.A. Avaliação de extratores para zinco e boro disponíveis em Latossolos do Triângulo Mineiro. R. Bras. Ci. Solo, 8:85-89, 1984.

SHUKLA, U.C. \& MITTAL, S.B. Characterization of zinc adsorption in some soils of India. Soil Sci. Soc. Am. J., 43:905-908, 1979.

SIMS, J.L. \& PATRICK Jr., W.H. The distribution of micronutrient cations in soil under conditions of varying redox potential and pH. Soil Sci. Soc. Am. J., 42:258-268, 1978.

SIMS, T.J. Soil $\mathrm{pH}$ effects on the distribution and plant availability of manganese, copper and zinc. Soil Sci. Soc. Am. J., 50:367-373, 1986.

STAHL, R.S. \& JAMES, B.R. Zinc sorption by iron oxides coated sand as a function of $\mathrm{pH}$. Soil Sci. Soc. Am. J., 55:1287-1290, 1991.

STEVENSON, F.J. Micronutrients soil tests. In: MORTVEDT, J.J.; COX, F.R.; SHUMAN, L.M. \& WELCH, R.M., eds. Micronutrients in agriculture. Madison, Soil Science Society of America, 1991. p.427-472.

TRAN, T.S. \& SIMARD, R.R. Mehlich III - Extractable elements. In: CARTER, M.R. Soil sampling and methods of analysis. Boca Raton, Canadian Society of Soil Science, 1993. p.43-47.

UREN, N.C. Forms, reactions, and availabilitity of nickel in soils. Adv. Agron., 48:141-203, 1992. 